\title{
Quantum ring with the Rashba spin-orbit interaction in the regime of strong light-matter coupling
}

\author{
V. K. Kozin, ${ }^{1,2}$ I. V. Iorsh, ${ }^{2}$ O. V. Kibis, ${ }^{3,1, *}$ and I. A. Shelykh ${ }^{1,2}$ \\ ${ }^{1}$ Science Institute, University of Iceland, Dunhagi 3, IS-107 Reykjavik, Iceland \\ ${ }^{2}$ ITMO University, Saint Petersburg 197101, Russia \\ ${ }^{3}$ Department of Applied and Theoretical Physics, Novosibirsk State Technical University, Karl Marx Avenue 20, Novosibirsk 630073, Russia
}

(Received 19 February 2018; revised manuscript received 5 April 2018; published 26 April 2018)

\begin{abstract}
We developed the theory of electronic properties of semiconductor quantum rings with the Rashba spin-orbit interaction irradiated by an off-resonant high-frequency electromagnetic field (dressing field). Within the Floquet theory of periodically driven quantum systems, it is demonstrated that the dressing field drastically modifies all electronic characteristics of the rings, including spin-orbit coupling, effective electron mass, and optical response. In particular, the present effect paves the way to controlling the spin polarization of electrons with light in prospective ring-shaped spintronic devices.
\end{abstract}

DOI: 10.1103/PhysRevB.97.155434

\section{INTRODUCTION}

The rapidly developing field of spintronics deals with spinrelated phenomena in mesoscopic transport [1-5]. Generally, the spins of individual carriers can be controlled either by application of an external magnetic field or via a change of the strength of the spin-orbit interaction (SOI) in the system. The second approach forms the basis of so-called nonmagnetic spintronics, which has attracted an enormous amount of interest in the scientific community. In particular, two mechanisms of the SOI are relevant for semiconductor structures: the Dresselhaus SOI [6], which was caused by the inversion asymmetry of the crystal lattice, and the Rashba SOI [7-10], which originated from the inversion asymmetry of the structure as a whole. The latter mechanism is of specific interest for spintronic applications since it becomes dominant in conventionally used InAs/GaSb-, AlSb/InAs-, and GaAs/GaAlAsbased nanostructures [11-13], and it can be easily tuned by an external gate voltage [14-16]. Recently, the alternative way of tuning SOI by purely optical methods was developed [17-19]. It is based on the regime of strong light-matter coupling when the system "electron + electromagnetic field" cannot be divided into weakly interacting optical and electronic subsystems. As a consequence, the hybrid electron-field object-the so-called "electron dressed by electromagnetic field" (dressed electron) - appears as an elementary quasiparticle [20,21]. The physical properties of dressed electrons can differ sufficiently from their "bare" counterparts, as was demonstrated for a wide variety of condensed-matter structures, including bulk semiconductors [22-24], quantum wells [25-29], quantum rings [30-35], graphene [36-44], topological insulators [45], etc. From the viewpoint of spintronic applications, it is crucially important that the SOI strength can be modified by laser irradiation [19] since this allows direct optical tuning of the spin relaxation time in a two-dimensional (2D) electron gas

\footnotetext{
*oleg.kibis@nstu.ru
}

[17], and therefore it paves the way to optically controlled spintronic devices [18].

Although the first ferromagnetic spintronic device (the Datta-Das spin transistor $[46,47]$ ) has been realized experimentally, its technological production remains challenging due to the difficulties with the efficient spin injection from ferromagnetic contacts. Therefore, the design of nonmagnetic spintronic devices, which do not require the presence of ferromagnetic elements, is still an actual problem. As a possible way to solve the problem, it was proposed to use semiconductor quantum rings (QRs) with the Rashba SOI, which induces the phase shift between spin waves propagating in the clockwise and counterclockwise directions. In turn, this results in the large conductance modulation due to the interference of the spin waves [48]. As a consequence, the physical basis of various QR-based nonferromagnetic spintronic devicesincluding spin transistors, spin filters, and quantum splittersappears [49-64]. In the aforementioned previous studies on the subject, the spin properties of QRs were assumed to be controlled by gate voltage. As to the optical methods of the spin control of QRs, they have escaped attention up to now. The present theoretical research aims partially to fill this gap in the spintronics of QRs.

The paper is organized as follows. In Sec. II, we derived the effective Hamiltonian of the irradiated QR with the Rashba spin-orbit interaction within the Floquet theory of periodically driven quantum systems. In Sec. III, the elaborated theory is applied to analyze spin and optical characteristics of the irradiated QR. Section IV contains our conclusions.

\section{MODEL}

To describe an irradiated QR (see Fig. 1), we have to start from the Hamiltonian describing an irradiated twodimensional (2D) electron system with the Rashba spin-orbit interaction [17]

$$
\hat{\mathcal{H}}_{2 \mathrm{D}}=\frac{(\hat{\mathbf{p}}-e \mathbf{A})^{2}}{2 m}+\alpha\left[\sigma_{x}\left(\hat{p}_{y}-e A_{y}\right)-\sigma_{y}\left(\hat{p}_{x}-e A_{x}\right)\right],
$$




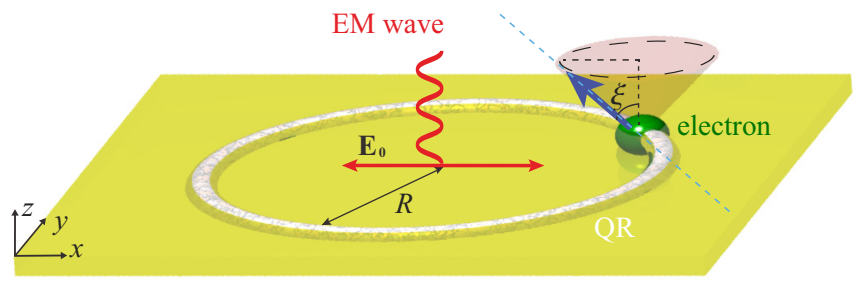

FIG. 1. Sketch of the system under consideration: The quantum ring $(\mathrm{QR})$ with the radius $R$ irradiated by a linearly polarized electromagnetic (EM) wave with the electric-field amplitude $E_{0}$. The electron spin (the dark blue arrow) is directed along the local quantization axis (the dashed blue line) with the spin angle $\xi$.

where $\hat{\mathbf{p}}=\left(\hat{p}_{x}, \hat{p}_{y}\right)$ is the operator of electron momentum, $m$ is the effective electron mass, $e$ is the electron charge, $\alpha$ is the Rashba spin-orbit coupling constant, $\sigma_{x, y, z}$ are the Pauli matrices, $\mathbf{A}=\left(A_{x}, A_{y}\right)=\left(\left[E_{0} / \omega\right] \cos \omega t, 0\right)$ is the vector potential of a linearly polarized electromagnetic wave (dressing field) in the 2D plane, $E_{0}$ is the electric field amplitude of the wave, and $\omega$ is the wave frequency, which is assumed to be far from resonant electron frequencies. Applying the standard approach [66] to transform the $2 \mathrm{D}$ electron system into the one-dimensional (1D) ring-shaped one, we arrive from the 2D Hamiltonian (1) at the Hamiltonian of an irradiated QR,

$$
\hat{\mathcal{H}}_{\mathrm{QR}}=\hat{\mathcal{H}}^{\prime}+\left[\sum_{n=1}^{2} \hat{V}_{n} e^{i n \omega t}+\text { H.c. }\right] \text {, }
$$

where the stationary part,

$$
\hat{\mathcal{H}}^{\prime}=\frac{\hat{l}_{z}^{2}}{2 m R^{2}}+\frac{\alpha}{R}\left[\sigma_{\rho} \hat{l}_{z}-i \hbar \frac{\sigma_{\varphi}}{2}\right]+\frac{e^{2} E_{0}^{2}}{4 m \omega^{2}},
$$

is the Hamiltonian of the unperturbed $\mathrm{QR}$ up to a field-induced constant shift of energy,

$$
\begin{gathered}
\hat{V}_{1}=\frac{e E_{0}}{2 m R \omega}\left(\sin \varphi \hat{l}_{z}-i \hbar \frac{\cos \varphi}{2}\right)+\frac{\alpha e E_{0}}{2 \omega} \sigma_{y}, \\
\hat{V}_{2}=\frac{e^{2} E_{0}^{2}}{8 m \omega^{2}},
\end{gathered}
$$

is the periodic part with the two harmonics originated from the irradiation, $R$ is the $\mathrm{QR}$ radius, $\hat{l}_{z}=-i \hbar \partial / \partial \varphi$ is the operator of angular momentum along the $z$ axis, $\varphi$ is the polar angle of an electron in the $\mathrm{QR}$, and $\sigma_{\rho}=\cos \varphi \sigma_{x}+\sin \varphi \sigma_{y}$ and $\sigma_{\varphi}=-\sin \varphi \sigma_{x}+\cos \varphi \sigma_{y}$ are the Pauli matrices written in polar coordinates. Applying the conventional Floquet-Magnus approach [67-69] to renormalize the Hamiltonian of an irradiated $\mathrm{QR}$ and restricting the consideration by the leading terms in the high-frequency limit, we can reduce the time-dependent Hamiltonian (2) to the effective time-independent one,

$$
\hat{\mathcal{H}}=\hat{\mathcal{H}}^{\prime}+\sum_{n=1}^{2} \frac{\left[\hat{V}_{n}, \hat{V}_{n}^{\dagger}\right]}{\hbar n \omega}+\sum_{n=1}^{2} \frac{\left[\left[\hat{V}_{n}, \hat{\mathcal{H}}^{\prime}\right], \hat{V}_{n}^{\dagger}\right]+\text { H.c. }}{2(\hbar n \omega)^{2}} .
$$

Substituting Eqs. (3)-(5) into Eq. (6), one can rewrite the effective Hamiltonian (6) as

$$
\hat{\mathcal{H}}=\hat{\mathcal{H}}_{0}+\hat{V},
$$

where

$$
\begin{aligned}
\hat{\mathcal{H}}_{0}= & \frac{\hat{l}_{z}^{2}}{2 m^{*} R^{2}}+\frac{\alpha}{R}\left[\sigma_{\rho} \hat{l}_{z}-i \hbar \frac{\sigma_{\varphi}}{2}\right]-\left(\frac{e E_{0} \alpha}{R \omega^{2}}\right)^{2} \frac{\hat{l}_{z} \sigma_{z}}{m \hbar} \\
& +\frac{e^{2} E_{0}^{2}}{4 m \omega^{2}}+\frac{1}{2 m}\left(\frac{\hbar e E_{0}}{4 m R^{2} \omega^{2}}\right)^{2}, \\
\hat{V}= & {\left[\frac{3}{16} \gamma_{1}^{2} \cos 2 \varphi-\gamma_{1}^{2} \gamma_{2}\left(\gamma_{2}^{2}-\frac{1}{4}\right) i \sigma_{x} \sin \varphi\right] \frac{\hbar^{2}}{2 m R^{2}} } \\
+ & {\left[\frac{i \gamma_{1}^{2} \sin 2 \varphi}{2}-2 \gamma_{1}^{2} \gamma_{2}\left(\gamma_{2}^{2}-\frac{1}{4}\right) \sigma_{x} \cos \varphi\right] \frac{\hbar \hat{l}_{z}}{2 m R^{2}} } \\
+ & \frac{\gamma_{1}^{2} \cos 2 \varphi}{8 m R^{2}} \hat{l}_{z}^{2},
\end{aligned}
$$

where

$$
m^{*}=\frac{m}{1+3\left(e E_{0} / 2 m R \omega^{2}\right)^{2}}
$$

is the effective electron mass renormalized by the irradiation, $\gamma_{1}=|e| E_{0} /\left(m R \omega^{2}\right)$ is the dimensionless parameter describing the strength of electron-field coupling, and $\gamma_{2}=m R \alpha / \hbar$ is the dimensionless parameter describing the strength of Rashba spin-orbit coupling. As expected, the Hamiltonian (7) exactly coincides with the Hamiltonian of an unirradiated QR [66] in the absence of the field $\left(E_{0}=0\right)$.

It should be noted that all effects that originated from the direct spin interaction with the magnetic component of the dressing field (particularly, the Zeeman effect and the Aharonov-Bohm effect) are relativistically negligible since the amplitude of magnetic induction of the field, $B_{0}=E_{0} / c$, is very small for reasonable field intensities. Therefore, they are omitted in the developed theory. We also neglected effects that arose from overlying electronic modes, assuming the typical distance between transverse electronic minibands (tens of meV for state-of-the-art QRs [65]) to be sufficiently larger than the photon and electron energies under consideration.

\section{RESULTS AND DISCUSSION}

To consider the Schrödinger problem with the effective Hamiltonian (7), let us start from its part (8). Two exact eigenstates of the Hamiltonian (8) can be written as

$$
\Psi_{1}(\varphi)=e^{i j_{z} \varphi}\left(\begin{array}{c}
\cos (\xi / 2) e^{-i \varphi / 2} \\
-\sin (\xi / 2) e^{i \varphi / 2}
\end{array}\right)
$$

and

$$
\Psi_{2}(\varphi)=e^{i j_{z} \varphi}\left(\begin{array}{c}
\sin (\xi / 2) e^{-i \varphi / 2} \\
\cos (\xi / 2) e^{i \varphi / 2}
\end{array}\right)
$$

where

$$
\xi=\arctan \left[\frac{2 m^{*} R \alpha / \hbar}{2\left(m^{*} / m\right)\left(e E_{0} \alpha / \omega^{2} \hbar\right)^{2}+1}\right]
$$

is the angle between the local spin quantization axis and the $z$ axis (see Fig. 1). It follows from single-valuedness of the eigenstates, $\Psi_{1,2}(\varphi)=\Psi_{1,2}(\varphi+2 \pi)$, that the $z$ component of total angular momentum of the electron, $j_{z}$, must satisfy the condition, $j_{z}=\lambda n+1 / 2$, where $n=0,1,2, \ldots$ is the orbital quantum number corresponding to the electron rotation in the 
$\mathrm{QR}$, and the sign $\lambda= \pm$ describes the direction of the rotation (counterclockwise/clockwise). Omitting constant terms that only shift the zero energy, one can write the electron energy spectrum of the eigenstates (11) and (12) as

$$
\begin{aligned}
\varepsilon_{\lambda n}^{s}= & \frac{\hbar^{2}}{2 m^{*} R^{2}}\left(\lambda n+\frac{1}{2}\right)^{2}+\frac{\hbar^{2}}{2 m^{*} R^{2}}\left|\lambda n+\frac{1}{2}\right| s \\
& \times \sqrt{\left[2\left(\frac{m^{*}}{m}\right)\left(\frac{e E_{0} \alpha}{\hbar \omega^{2}}\right)^{2}+1\right]^{2}+\left[\frac{2 \alpha m^{*} R}{\hbar}\right]^{2}},
\end{aligned}
$$

where $s= \pm 1$ is the quantum number describing the spin direction along the local quantization axis (see Fig. 1), and the spin $s=+1$ corresponds to the greater energy (14). Within the conventional notation [70] based on the three quantum numbers, $|n, \lambda, s\rangle$, the eigenstates (11) and (12) can be written as

$$
\begin{gathered}
|n,+,-1\rangle=e^{i n \varphi}\left(\begin{array}{c}
\cos (\xi / 2) \\
-\sin (\xi / 2) e^{i \varphi}
\end{array}\right), \\
|n,+,+1\rangle=e^{i n \varphi}\left(\begin{array}{c}
\sin (\xi / 2) \\
\cos (\xi / 2) e^{i \varphi}
\end{array}\right), \\
|n,-,+1\rangle=e^{-i n \varphi}\left(\begin{array}{c}
\cos (\xi / 2) \\
-\sin (\xi / 2) e^{i \varphi}
\end{array}\right), \\
|n,-,-1\rangle=e^{-i n \varphi}\left(\begin{array}{c}
\sin (\xi / 2) \\
\cos (\xi / 2) e^{i \varphi}
\end{array}\right)
\end{gathered}
$$

for $n=1,2,3, \ldots$ and

$$
\begin{aligned}
|0,+,-1\rangle & =\left(\begin{array}{c}
\cos (\xi / 2) \\
-\sin (\xi / 2) e^{i \varphi}
\end{array}\right), \\
|0,+,+1\rangle & =\left(\begin{array}{c}
\sin (\xi / 2) \\
\cos (\xi / 2) e^{i \varphi}
\end{array}\right)
\end{aligned}
$$

for $n=0$. It follows from Eq. (14), in particular, that $\varepsilon_{-n}^{s}=$ $\varepsilon_{n-1}^{s}$. This means that the states $|n,-, s\rangle$ and $|n-1,+, s\rangle$ are degenerated.

The eigenstates and eigenenergies (11)-(20) can be easily verified by direct substitution into the Schrödinger equation with the Hamiltonian (8). However, the total effective Hamiltonian (7) consists of the two parts, including both the discussed Hamiltonian $\hat{\mathcal{H}}_{0}$ and the term $\hat{V}$. Therefore, we have to analyze the effect of the term $\hat{V}$ on the found solutions of the Schrödinger problem with the Hamiltonian $\hat{\mathcal{H}}_{0}$. It follows from Eqs. (9) and (15)-(20) that $\left\langle n^{\prime}, \lambda^{\prime}, s^{\prime}|\hat{V}| n, \lambda, s\right\rangle \sim \delta_{\lambda^{\prime} \lambda}$ for $n, n^{\prime} \geqslant 1$. Thus, the term $\hat{V}$ does not split the degenerate states $|n,-, s\rangle$ and $|n-1,+, s\rangle$. It should be noted also that the discussed regime of strong light-matter coupling is conventionally defined as a light-induced renormalization of electronic properties without the light absorption by electrons (see, e.g., the discussion in Ref. [29]). Particularly, the main absorption mechanism for semiconductor structures dressed by an offresonant electromagnetic field - the collisional absorption of the field by conduction electrons-can be neglected if $\omega \tau \gg 1$, where $\tau$ is the electron relaxation time [28]. Therefore, we have to consider the case of high frequencies, $\omega$, when the condition $\gamma_{1} \ll 1$ can take place. It follows from this that the discussed term $\hat{V} \sim \gamma_{1}^{2}$ can be considered as a weak perturbation for a broad range of $\mathrm{QR}$ parameters. In particular, the conventional
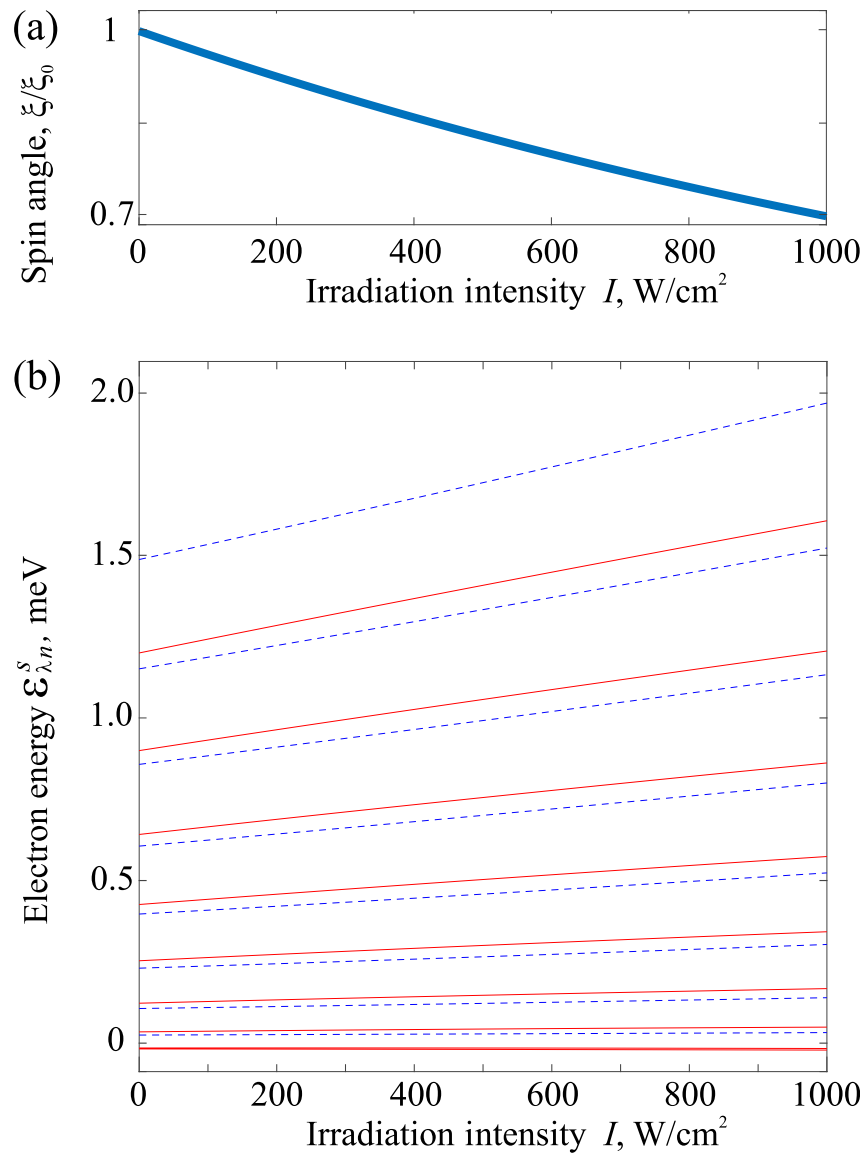

FIG. 2. Electronic characteristics of InGaAs-based QR (the electron effective mass is $m=0.045 m_{0}$, the Rashba coupling constant is $\alpha=10^{4} \mathrm{~m} / \mathrm{s}$, and the QR radius is $R=200 \mathrm{~nm}$ ) irradiated by a dressing field with the frequency $\omega=1.6 \times 10^{12} \mathrm{rad} / \mathrm{s}$ : (a) Dependence of the spin angle, $\xi$, on the irradiation intensity, $I$; (b) dependence of the first nine electron energy levels, $\varepsilon_{\lambda n}^{s}$, on the irradiation intensity, $I$, for the counterclockwise electron rotation in the ring $(\lambda=+)$, where the dashed and solid lines correspond to different spin orientations $(s= \pm 1)$.

criterion of perturbation theory,

$$
\left|\frac{\left\langle n^{\prime}, \lambda^{\prime}, s^{\prime}|\hat{V}| n, \lambda, s\right\rangle}{\varepsilon_{\lambda^{\prime} n^{\prime}}^{s^{\prime}}-\varepsilon_{\lambda n}^{s}}\right| \ll 1,
$$

can be satisfied for the first tens of energy levels (14) in the typical case of InGaAs-based QRs with the effective mass $m=0.045 m_{e}$, radius $R \approx 200 \mathrm{~nm}$, and the Rashba coupling constant $\alpha \approx 10^{4} \mathrm{~m} / \mathrm{s}$. As a consequence, the effective Hamiltonian (7) can be reduced to the simplified Hamiltonian (8). Correspondingly, the found eigenstates and eigenenergies (11)-(20) can be applied to describe electronic properties of the irradiated $Q R$.

It follows from the Hamiltonian (8) that the irradiation of the QR results in two main effects: First, it renormalizes the electron effective mass (10), and second it leads to the unusual spin-orbit coupling $\sim l_{z} \sigma_{z}$ described by the third term of the Hamiltonian (8). In turn, these effects lead to the dependencies of the spin angle (13) and the energy levels (14) on the irradiation intensity, which are plotted in Fig. 2. It follows from Fig. 2(a) that the irradiation has a very strongly effect on 
the spin angle (13). Namely, the relatively weak irradiation can decrease the angle to tens of percent of its initial value in the unirradiated QR, $\xi_{0}=\arctan (2 \alpha m R / \hbar)$. Since the modulation of spin orientation by various external actions lies in the core of modern spintronics [1-4], the found strong dependence of the spin polarization on the irradiation can be used, particularly in prospective ring-shaped spintronic devices operated by light. It follows from Fig. 2(b) that the irradiation also strongly effects the energy of the electron levels in the QR and their spin splitting. Such a light-induced modification of the energy spectrum (14) can manifest itself, particularly in the optical measurements discussed below.

Let us consider a QR irradiated by a two-mode electromagnetic wave consisting of a strong dressing field (which renormalizes the energy spectrum of electrons according to what was mentioned earlier) and a relatively weak probe field with the frequency $\Omega$ (which can detect the discussed renormalization of the energy spectrum). The optical spectrum of absorption of the probe field can be obtained with use of the conventional Kubo formalism [71]. Within this approach, the longitudinal conductivity describing the response of the $\mathrm{QR}$ to the probe field polarized along the $j=x, y$ axis reads

$$
\sigma_{j j}=\sum_{\substack{n, \lambda, s \\ n^{\prime}, \lambda^{\prime}, s^{\prime}}} \frac{\left[f\left(\varepsilon_{\lambda^{\prime} n^{\prime}}^{s^{\prime}}\right)-f\left(\varepsilon_{\lambda n}^{s}\right)\right]\left|\left\langle n^{\prime}, \lambda^{\prime}, s^{\prime}\left|\hat{v}_{j}\right| n, \lambda, s\right\rangle\right|^{2}}{\left(\varepsilon_{\lambda^{\prime} n^{\prime}}^{s^{\prime}}-\varepsilon_{\lambda n}^{s}\right)\left(\varepsilon_{\lambda^{\prime} n^{\prime}}^{s^{\prime}}-\varepsilon_{\lambda n}^{s}+\hbar \Omega+i \Gamma\right)} \frac{\hbar e^{2}}{i \pi R^{2}},
$$

where $f(\varepsilon)$ is the Fermi-Dirac distribution function, $\hat{v}_{j}=$ $\hat{p}_{j} / m$ is the velocity operator, and $\Gamma=\hbar / \tau$ is the broadening of energy levels depending on the electron relaxation time, $\tau$. It should be noted that the used spin index, $s= \pm 1$, describes the spin projection on the local quantization axis (see the dashed line in Fig. 1), which depends on the electron location in the QR and, correspondingly, on the direction of the vector of electron velocity. As a consequence, the matrix of the velocity operator in Eq. (22), $\left\langle n^{\prime}, \lambda^{\prime}, s^{\prime}\left|\hat{v}_{j}\right| n, \lambda, s\right\rangle$, is not diagonal in this spin index. In particular, direct calculation results in $\left\langle n^{\prime}, \pm, s^{\prime}\left|\hat{v}_{j}\right| n, \pm, s\right\rangle \sim\left(\delta_{n-n^{\prime}, 1}+\delta_{n-n^{\prime},-1}\right)$ and $\left\langle n^{\prime}, \mp\right.$ $\left., s^{\prime}\left|\hat{v}_{j}\right| n, \pm, s\right\rangle \sim \delta_{n+n^{\prime}, 1}$. As a consequence, the probe field can induce electron transitions between the electron states with mutually opposite local spin directions. Substituting Eqs. (14)(20) into Eq. (22), one can calculate the sought-after absorption spectrum of the probe field (see Fig. 3), which is represented by the real part of the conductivity, $\operatorname{Re}\left(\sigma_{j j}\right)$. In the absence of the dressing field, the absorption spectrum of the QR plotted in Fig. 3(a) consists of the three peaks corresponding to the following electron transitions: $|5,+,+1\rangle \rightarrow|4,+,+1\rangle$, $|7,+,-1\rangle \rightarrow|6,+,+1\rangle$, and $|7,+,-1\rangle \rightarrow|6,+,-1\rangle$ (peak 1); $|6,+,+1\rangle \rightarrow|5,+,+1\rangle,|8,+,-1\rangle \rightarrow \mid 7,+$ $, 1\rangle$, and $|8,+,-1\rangle \rightarrow|7,+,-1\rangle($ peak 2$) ;|7,+,+1\rangle \rightarrow$ $|6,+,+1\rangle$ and $|9,+,-1\rangle \rightarrow|8,+,-1\rangle$ (peak 3).

The evolution of this spectrum under the influence of the dressing field is presented in Figs. 3(b)-3(d). In the absence of the dressing field, the highest peak 3 originates from the transitions $|6,+,+1\rangle \rightarrow|5,+,+1\rangle$ and $|8,+,-1\rangle \rightarrow$ $|7,+,-1\rangle$ since the chosen Fermi energy, $\mu=1 \mathrm{meV}$, lies in the middle between the corresponding levels [see Fig. 2(b)]. Since the dressing field increases the distance between the energy levels (14), it shifts the peaks to the right and deforms
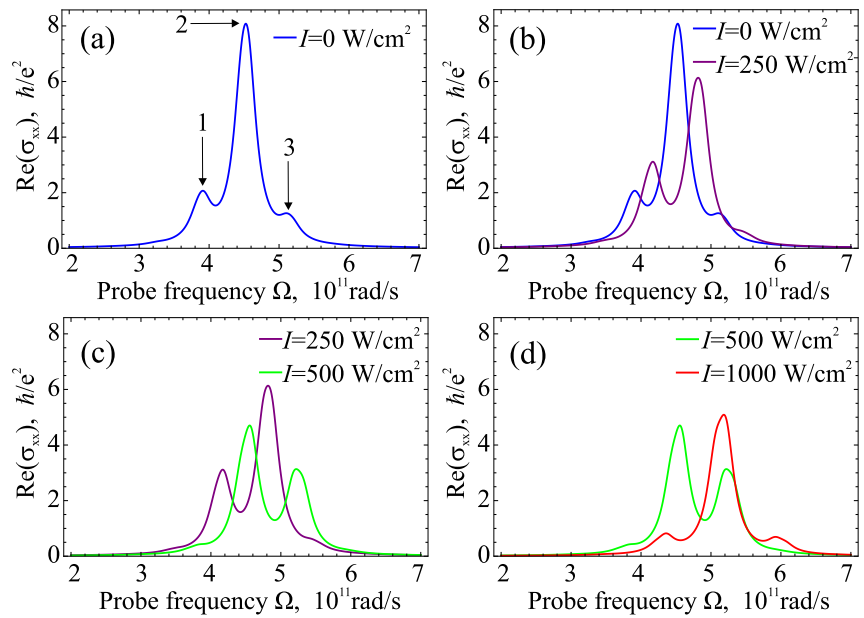

FIG. 3. Absorption spectra of the probe field with the frequency $\Omega$ for the InGaAs-based QR (the electron effective mass is $m=$ $0.045 m_{0}$, the Rashba coupling constant is $\alpha=10^{4} \mathrm{~m} / \mathrm{s}$, the electron relaxation time is $\tau=70 \mathrm{ps}$, the temperature is $T=5 \mathrm{~K}$, the Fermi energy is $\mu=1 \mathrm{meV}$, and the QR radius is $R=200 \mathrm{~nm}$ ) irradiated by a dressing field with the frequency $\omega=1.6 \times 10^{12} \mathrm{rad} / \mathrm{s}$ and different irradiation intensities, $I$.

them [see Figs. 3(b)-3(d)]. It should be noted that the shape of the spectrum at the irradiation intensity $I=1000 \mathrm{~W} / \mathrm{cm}^{2}$ is very similar to case of an unirradiated QR [compare Figs. 3(a) and 3(d)]. Physically, the similarity appears since the Fermi energy, $\mu=1 \mathrm{meV}$, lies at this intensity again in the middle between the corresponding levels [see Fig. 2(b)]. However, the highest peak in this case arises from the peak 1 in Fig. 3(a), and therefore it corresponds to the transitions $|5,+,+1\rangle \rightarrow$ $|4,+,+1\rangle,|7,+,-1\rangle \rightarrow|6,+,+1\rangle$, and $|7,+,-1\rangle \rightarrow$ $|6,+,-1\rangle$. Finalizing the discussion, let us formulate how the dressing field parameters should be chosen in experiments. It follows from the Hamiltonian (8) that the absolute value of the light-induced renormalization of all electronic characteristics is proportional to the squared ratio $E_{0} / \omega^{2}$. Therefore, we have to keep this ratio not too small to observe the discussed renormalization experimentally for reasonable dressing field amplitudes, $E_{0}$. This is why the dressing field frequency, $\omega$, in Figs. 2 and 3 is chosen to be in the THz range.

\section{CONCLUSIONS}

In conclusion, we demonstrated that the key electronic characteristics of QRs with the Rashba spin-orbit interaction the structure of electron energy levels and the spin polarization of electrons - strongly depend on an off-resonant irradiation. In particular, the modification of both electron effective mass and spin-orbit coupling appears. It is shown that the irradiationinduced renormalization of the electron energy spectrum can be observed in state-of-the-art optical experiments, whereas the light sensitivity of the spin orientation can be exploited in prospective spintronic devices operated by light.

\section{ACKNOWLEDGMENTS}

The work was partially supported by the RISE Program (project CoExAN), the Russian Foundation for Basic Research 
(Project No. 17-02-00053), Rannis project 163082-051, Ministry of Education and Science of Russian Federation (Projects No. 3.4573.2017/6.7, No. 3.2614.2017/4.6,
No. 3.1365.2017/4.6, No. 3.8884.2017/8.9, and No. 14.Y26.31.0015), and Government of Russian Federation (Grant No. 08-08).
[1] D. D. Awschalom, D. Loss, and N. Samarth, Semiconductor Spintronics and Quantum Computation (Springer-Verlag, Berlin, 2002).

[2] I. Zutic, J. Fabian, and S. D. Sarma, Spintronics: Fundamentals and applications, Rev. Mod. Phys. 76, 323 (2004).

[3] D. D. Awschalom and M. E. Flatté, Challenges for semiconductor spintronics, Nat. Phys. 3, 153 (2007).

[4] M. Cahay, Spin transistors: Closer to an all-electric device, Nat. Nanotechnol. 10, 21 (2015).

[5] V. Szaszko-Bogar, F. M. Peeters, and P. Földi, Oscillating spinorbit interaction in two-dimensional superlattices: Sharp transmission resonances and time-dependent spin-polarized currents, Phys. Rev. B 91, 235311 (2015).

[6] G. Dresselhaus, Spin-orbit coupling effects in zinc blende structures, Phys. Rev. 100, 580 (1955).

[7] E. I. Rashba, Properties of semiconductors with an extremum loop. 1. Cyclotron and combinational resonance in a magnetic field perpendicular to the plane of the loop, Sov. Phys. Solid State 2, 1109 (1960).

[8] Y. A. Bychkov and E. I. Rashba, Oscillatory effects and the magnetic susceptibility of carriers in inversion layers, J. Phys. C 17, 6039 (1984).

[9] D. Bercioux and P. Lucignano, Quantum transport in Rashba spin-orbit materials: A review, Rep. Prog. Phys. 78, 106001 (2015).

[10] R. Winkler, Spin-Orbit Coupling Effects in Two-Dimensional Electron and Hole Systems (Springer-Verlag, Berlin, 2003).

[11] B. Miller, D. M. Zumbuhl, C. M. Marcus, Y. B. Lyanda-Geller, D. Goldhaber-Gordon, K. Campman, and A. C. Gossard, GateControlled Spin-Orbit Quantum Interference Effects in Lateral Transport, Phys. Rev. Lett. 90, 076807 (2003).

[12] A. Studenikin, P. T. Coleridge, N. Ahmed, P. Poole and A. Sachrajda, Experimental study of weak antilocalization effects in a high-mobility $\operatorname{In}_{x} \mathrm{Ga}_{1-x}$ As/InP quantum well, Phys. Rev. B 68, 035317 (2003).

[13] A. Ghosh, C. J. B. Ford, M. Pepper, H. E. Beere, and D. A. Ritchie, Possible Evidence of a Spontaneous Spin Polarization in Mesoscopic Two-Dimensional Electron Systems, Phys. Rev. Lett. 92, 116601 (2004).

[14] J. Nitta, T. Akazaki, H. Takayanagi, and T. Enoki, Gate Control of Spin-Orbit Interaction in an Inverted $\mathrm{In}_{0.53} \mathrm{Ga}_{0.47} \mathrm{As} / \mathrm{In}_{0.52} \mathrm{Al}_{0.48}$ As Heterostructure, Phys. Rev. Lett. 78, 1335 (1997).

[15] G. Engels, J. Lange, Th. Schäpers, and H. Lüth, Experimental and theoretical approach to spin splitting in modulation-doped $\mathrm{In}_{x} \mathrm{Ga}_{1-x} \mathrm{As} / \mathrm{InP}$ quantum wells for $\mathrm{B} \rightarrow 0$, Phys. Rev. B 55, R1958 (1997).

[16] J. P. Heida, B. J. van Wees, J. J. Kuipers, T. M. Klapwijk, and G. Borghs, Spin-orbit interaction in a two-dimensional electron gas in a InAs/AlSb quantum well with gate-controlled electron density, Phys. Rev. B 57, 11911 (1998).

[17] A. A. Pervishko, O. V. Kibis, S. Morina, and I. A. Shelykh, Control of spin dynamics in a two-dimensional electron gas by electromagnetic dressing, Phys. Rev. B 92, 205403 (2015).
[18] A. S. Sheremet, O. V. Kibis, A. V. Kavokin, and I. A. Shelykh, Datta-and-Das spin transistor controlled by a high-frequency electromagnetic field, Phys. Rev. B 93, 165307 (2016).

[19] D. Yudin and I. A. Shelykh, Two-dimensional electron gas in the regime of strong light-matter coupling: Dynamical conductivity and all-optical measurements of Rashba and Dresselhaus coupling, Phys. Rev. B 94, 161404(R) (2016).

[20] C. Cohen-Tannoudji, J. Dupont-Roc, and G. Grynberg, AtomPhoton Interactions: Basic Processes and Applications (Wiley, Chichester, 1998).

[21] M. O. Scully and M. S. Zubairy, Quantum Optics (Cambridge University Press, Cambridge, 2001).

[22] S. P. Goreslavskii and V. F. Elesin, Electric properties of a semiconductor in the field of a strong electromagnetic wave, JETP Lett. 10, 316 (1969).

[23] Q. T. Vu, H. Haug, O. D. Mucke, T. Tritschler, M. Wegener, G. Khitrova, and H. M. Gibbs, Light-Induced Gaps in Semiconductor Band-To-Band Transitions, Phys. Rev. Lett. 92, 217403 (2004).

[24] Q. T. Vu and H. Haug, Detection of light-induced band gaps by ultrafast femtosecond pump and probe spectroscopy, Phys. Rev. B 71, 035305 (2005).

[25] A. Mysyrowicz, D. Hulin, A. Antonetti, A. Migus, W. T. Masselink, and H. Morkoc, "Dressed Excitons" in a MultipleQuantum-Well Structure: Evidence for an Optical Stark Effect with Femtosecond Response Time, Phys. Rev. Lett. 56, 2748 (1986).

[26] M. Wagner, H. Schneider, D. Stehr, S. Winnerl, A. M. Andrews, S. Schartner, G. Strasser, and M. Helm, Observation of the Intraexciton Autler-Townes Effect in GaAs/AlGaAs Semiconductor Quantum Wells, Phys. Rev. Lett. 105, 167401 (2010).

[27] M. Teich, M. Wagner, H. Schneider, and M. Helm, Semiconductor quantum well excitons in strong, narrowband terahertz fields, New J. Phys. 15, 065007 (2013).

[28] O. V. Kibis, How to suppress the backscattering of conduction electrons? Europhys. Lett. 107, 57003 (2014).

[29] S. Morina, O. V. Kibis, A. A. Pervishko, and I. A. Shelykh, Transport properties of a two-dimensional electron gas dressed by light, Phys. Rev. B 91, 155312 (2015).

[30] O. V. Kibis, Dissipationless Electron Transport in PhotonDressed Nanostructures, Phys. Rev. Lett. 107, 106802 (2011).

[31] F. K. Joibari, Y. M. Blanter, and G. E. W. Bauer, Light-induced spin polarizations in quantum rings, Phys. Rev. B 90, 155301 (2014).

[32] H. Sigurdsson, O. V. Kibis, and I. A. Shelykh, Optically induced Aharonov-Bohm effect in mesoscopic rings, Phys. Rev. B 90, 235413 (2014).

[33] O. V. Kibis, H. Sigurdsson, and I. A. Shelykh, Aharonov-Bohm effect for excitons in a semiconductor quantum ring dressed by circularly polarized light, Phys. Rev. B 91, 235308 (2015).

[34] M. Hasan, I. V. Iorsh, O. V. Kibis, and I. A. Shelykh, Optically controlled periodical chain of quantum rings, Phys. Rev. B 93 , 125401 (2016). 
[35] V. K. Kozin, I. V. Iorsh, O. V. Kibis, and I. A. Shelykh, Periodic array of quantum rings strongly coupled to circularly polarized light as a topological insulator, Phys. Rev. B 97, 035416 (2018).

[36] F. J. Lopez-Rodriguez and G. G. Naumis, Analytic solution for electrons and holes in graphene under electromagnetic waves: Gap appearance and nonlinear effects, Phys. Rev. B 78, 201406(R) (2008).

[37] T. Oka and H. Aoki, Photovoltaic Hall effect in graphene, Phys. Rev. B 79, 081406(R) (2009).

[38] O. V. Kibis, Metal-insulator transition in graphene induced by circularly polarized photons, Phys. Rev. B 81, 165433 (2010).

[39] T. Kitagawa, T. Oka, A. Brataas, L. Fu, and E. Demler, Transport properties of nonequilibrium systems under the application of light: Photoinduced quantum Hall insulators without Landau levels, Phys. Rev. B 84, 235108 (2011).

[40] G. Usaj, P. M. Perez-Piskunow, L. E. F. Foa Torres, and C. A. Balseiro, Irradiated graphene as a tunable Floquet topological insulator, Phys. Rev. B 90, 115423 (2014).

[41] K. Kristinsson, O. V. Kibis, S. Morina, and I. A. Shelykh, Control of electronic transport in graphene by electromagnetic dressing, Sci. Rep. 6, 20082 (2016).

[42] O. V. Kibis, S. Morina, K. Dini, and I. A. Shelykh, Magnetoelectronic properties of graphene dressed by a high-frequency field, Phys. Rev. B 93, 115420 (2016).

[43] O. V. Kibis, K. Dini, I. V. Iorsh, and I. A. Shelykh, All-optical band engineering of gapped Dirac materials, Phys. Rev. B 95, 125401 (2017).

[44] I. V. Iorsh, K. Dini, O. V. Kibis, and I. A. Shelykh, Optically induced Lifshitz transition in bilayer graphene, Phys. Rev. B 96, 155432 (2017).

[45] N. H. Lindner, G. Refael, and V. Galitski, Floquet topological insulator in semiconductor quantum wells, Nat. Phys. 7, 490 (2011).

[46] S. Datta and B. Das, Electronic analog of the electro-optic modulator, Appl. Phys. Lett. 56, 665 (1990).

[47] H. C. Koo, J. H. Kwon, J. Eom, J. Chang, S. H. Han, and M. Johnson, Control of spin precession in a spin-injected field effect transistor, Science 325, 1515 (2009).

[48] T. Bergsten, T. Kobayashi, Y. Sekine, and J. Nitta, Experimental Demonstration of the Time Reversal Aharonov-Casher Effect, Phys. Rev. Lett. 97, 196803 (2006).

[49] J. Nitta, F. E. Meijer, and H. Takayanagi, Spin-interference device, Appl. Phys. Lett. 75, 695 (1999).

[50] J. Nitta and T. Koga, Rashba spin-orbit interaction and its applications to spin-interference effect and spin-filter device, J. Supercond. 16, 689 (2003).

[51] D. Frustaglia and K. Richter, Spin interference effects in ring conductors subject to Rashba coupling, Phys. Rev. B 69, 235310 (2004).

[52] I. A. Shelykh, N. T. Bagraev, N. G. Galkin, and L. E. Klyachkin, Interplay of $h / e$ and $h / 2 e$ oscillations in gatecontrolled Aharonov-Bohm rings, Phys. Rev. B 71, 113311 (2005).

[53] A. G. Aronov and Y. B. Lyanda-Geller, Spin-Orbit Berry Phase in Conducting Rings, Phys. Rev. Lett. 70, 343 (1993).
[54] M. Popp, D. Frustaglia, and K. Richter, Spin filter effects in mesoscopic ring structures, Nanotechnology 14, 347 (2003).

[55] A. A. Kiselev and K. W. Kim, T-shaped spin filter with a ring resonator, J. Appl. Phys. 94, 4001 (2003).

[56] I. A. Shelykh, N. G. Galkin, and N. T. Bagraev, Quantum splitter controlled by Rashba spin-orbit coupling, Phys. Rev. B 72 , 235316 (2005)

[57] B. Mòlnar, F. M. Peeters, and P. Vasilopoulos, Spin-dependent magnetotransport through a ring due to spin-orbit interaction, Phys. Rev. B 69, 155335 (2004).

[58] P. Földi, B. Mòlnar, M. G. Benedict, and F. M. Peeters, Spintronic single-qubit gate based on a quantum ring with spin-orbit interaction, Phys. Rev. B 71, 033309 (2005).

[59] P. Földi, O. Kálmán, M. G. Benedict, and F. M. Peeters, Quantum rings as electron spin beam splitters, Phys. Rev. B 73, 155325 (2006).

[60] R. Citro, F. Romeo, and M. Marinaro, Zero-conductance resonances and spin filtering effects in ring conductors subject to Rashba coupling, Phys. Rev. B 74, 115329 (2006).

[61] N. Hatano, R. Shirasaki, and H. Nakamura, Non-Abelian gauge field theory of the spin-orbit interaction and a perfect spin filter, Phys. Rev. A 75, 032107 (2007).

[62] S. Matityahu, A. Aharony, O. Entin-Wohlman, and S. Katsumoto, Robustness of spin filtering against current leakage in a Rashba-Dresselhaus-Aharonov-Bohm interferometer, Phys. Rev. B 87, 205438 (2013).

[63] S. Matityahu, A. Aharony, O. Entin-Wohlman, and S. Tarucha, Spin filtering in a Rashba-Dresselhaus-AharonovBohm double-dot interferometer, New J. Phys. 15, 125017 (2013).

[64] S. Matityahu, A. Aharony, O. Entin-Wohlman, and C. A. Balseiro, Spin filtering in all-electrical three-terminal interferometers, Phys. Rev. B 95, 085411 (2017).

[65] A. Lorke, J. R. Luyken, A. O. Govorov, J. P. Kotthaus, J. M. Garcia, and P. M. Petroff, Spectroscopy of Nanoscopic Semiconductor Rings, Phys. Rev. Lett. 84, 2223 (2000).

[66] F. E. Meijer, A. F. Morpurgo, and T. M. Klapwijk, Onedimensional ring in the presence of Rashba spin-orbit interaction: Derivation of the correct Hamiltonian, Phys. Rev. B 66, 033107 (2002).

[67] F. Casas, J. A. Oteo, and J. Ros, Floquet theory: Exponential perturbative treatment, J. Phys. A 34, 3379 (2001).

[68] N. GoldMan and J. Dalibard, Periodically Driven Quantum Systems: Effective Hamiltonians and Engineered Gauge Fields, Phys. Rev. X 4, 031027 (2014).

[69] A. Eckardt and E. Anisimovas, High-frequency approximation for periodically driven quantum systems from a Floquet-space perspective, New J. Phys. 17, 093039 (2015).

[70] F. Nagasawa, D. Frustaglia, H. Saarikoski, K. Richter, and J. Nitta, Control of the spin geometric phase in a semiconductor quantum ring, Nat. Commun. 4, 2526 (2013).

[71] H. Bruuc and K. Flensberg, Many-Body Quantum Theory in Condensed Matter Physics (Oxford University Press, Oxford, 2001). 